\title{
Article \\ Characterization of the pVHL interactome in human testis using high-throughput library screening
}

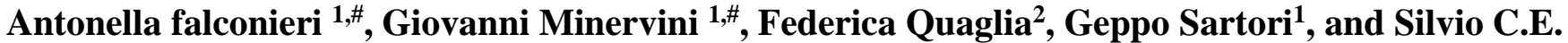 \\ Tosatto ${ }^{1, *}$ \\ Department of Biomedical Sciences, University of Padova, 35121 Padova, Italy \\ Institute of Biomembranes, Bioenergetics and Molecular Biotechnologies, National Research Council (CNR-IBIOM), \\ 70126, Bari, Italy \\ \# These authors contributed equally \\ * Correspondence: silvio.tosatto@unipd.it;
}

\begin{abstract}
Simple summary: The von Hippel-Lindau (pVHL) tumor suppressor is a protein that regulates the normal cell adaptation to low oxygen concentrations. When its function is altered by inherited or acquired mutation pVHL becomes causative of a familiar predisposition to develop different types of cancers. Besides this role, $\mathrm{pVHL}$ is also thought to have other relevant cell functions and studies in mouse demonstrated that this protein is crucial for the correct testis development and sperm maturation. By scanning testis-specific library, we identified 55 novel proteins that interact with the human pVHL, with many of them directly participating in metabolic pathways frequently altered in cancer. Furthermore, our results suggest that pVHL may be also important for correct gonads function in men.
\end{abstract}

\begin{abstract}
Functional impairment of the von Hippel-Lindau (pVHL) tumor suppressor is causative of a familiar increased risk to develop cancer. As E3 substrate recognition particle, pVHL marks for degradation the hypoxia inducible factor $1 \alpha$ (HIF-1 $\alpha$ ) in normoxic conditions, thus acting as a key regulator of both acute and chronic cell adaptation to hypoxia. Further evidence showed pVHL to also play relevant roles in microtubules stabilization, participate in the formation of the extracellular matrix, as well as to regulate cell senescence and apoptosis. Male mice model carrying VHL gene conditional knockout present significative abnormalities in testis development paired with defects in spermatogenesis and infertility, indicating that pVHL exerts testis-specific roles, at least in mice. Here, we describe 55 novel interactors of the human pVHL obtained by testis-tissue library screening. We show that pVHL interacts with multiple human proteins directly involved in spermatogenesis and reproductive metabolism, suggesting that, in addition to its role in cancer formation, pVHL may be pivotal in the correct gonads development also in human.
\end{abstract}

Keywords: VHL; von Hippel-Lindau syndrome; ubiquitination; hypoxia; testis; yeast two-hybrid (Y2H) 


\section{Introduction}

The von Hippel-Lindau (VHL) disease (OMIM number 193300) is a rare autosomal dominantly inherited genetic disorder [1] with an incidence of 1:36000 live births and high penetrance of $>90 \%$ by age 65 years with a mean age at tumor diagnosis of 26 years [2]. The VHL is a multiple-neoplasia disorder associated with the development of highly vascular tumors such as retinal and CNS hemangioblastoma (HBs), clear cell renal cell carcinoma (RCC), pheochromocytoma (PCC) and paraganglioma (PGL), pancreatic cystadenomas, endolymphatic sac tumors (ELSTs), epididymal cysts and broad ligament cystadenoma [3]. Clinical VHL manifestations are variable, with different phenotypes associated with the same mutation in different families or even in a same family [3]. The disease arises from pathogenic inactivation of the homonymous VHL gene located on the short arm of chromosome 3 (3p25-26) [4]. In humans, VHL encodes two protein products, a $30 \mathrm{kDa}$ full-length protein named pVHL30 and a shorter isoform referred as pVHL19 that is generated by an alternative translation initiation site at methionine 54 [5]. A third isoform, known as pVHL172, arises from an alternative spliced mRNA in which the exon E2 is excluded [6]. Full-length pVHL30 presents three distinct domains: an intrinsically disordered N-terminal tail (aa1-53), a central full $\beta$-domain (aa54 to 157) and a C-terminal $\alpha$-domain (aa158 to 213) $[7,8]$. In normoxic conditions, pVHL controls degradation of the hypoxia inducible factor $1 \alpha$ (HIF-1 $\alpha$ ) [7] acting as the substrate recognition particle of an ubiquitin ligase (E3) multiprotein complex, formed with the Elongin B, Elongin C, Cullin2 and Rbx1 $[9,10]$. Interaction of pVHL with HIF- $1 \alpha$ is highly specific and triggered by HIF$1 \alpha$ proline hydroxylation mediated by the prolyl-4 hydroxylase domain containing enzymes (PHD1, -2 , and -3 ), while it is inhibited under hypoxic conditions [7,11]. Apart from its role in degrading HIF- $1 \alpha$, multiple alternative functions were linked to pVHL such as to promote microtubules stabilization, regulate kinases activity, participate the formation of the extracellular matrix, as well as to regulate cell senescence and apoptosis [12-16]. The pVHL is frequently described as a molecular hub that mediates the interactions with more than 500 binding partners involved in multiple cell pathways $[17,18]$. The biological meaning of most of these interactions remains, however, poorly understood. Male mice carrying VHL conditional knockout present abnormalities in testis development, reduced sperm count, impaired spermatogenesis and infertility, besides altered vascularization in multiple organs which is a conventional marker of nonfunctional pVHL [19]. The $25 \%-60 \%$ of VHL male patients develop epididymal papillary cystadenomas [20,21], an otherwise rare form of neoplasm affecting male reproductive organs [22]. In contrast with the VHL murine model, no infertility or defect of spermatogenesis are reported in humans except for mechanical obstruction of seminal ducts due to epididymal cysts. On the other hand, pVHL is required for the correct cilia formation [23], while it is well understood that multiple male infertility syndromes arise from congenital defects in ciliogenesis [24]. Similarly, defects in ciliogenesis are also reported in cancer and linked to deregulation of the cellular response to signals from multiple pathways $[25,26]$.

Here, we describe the interactome around the human pVHL obtained through testistissue library screening by yeast mating. We identified 55 novel testis-specific pVHL30 interactors, with multiple proteins directly involved in spermatogenesis and reproductive metabolism, as well as playing a role in cancer formation.

\section{Materials and Methods}

\subsection{Bait vector construction}

The cDNA encoding pVHL30 was cloned into pGBKT7 vector using in vitro InFusion ${ }^{\circledR}$ method. The full-length cDNA encoding the human pVHL30 was transferred from pCDNA3.1 purchased by GenScript (GenEZ plasmid OHu23297) to pGBKT7 empty vector (Clontech). The cDNA was amplified by PCR using specific primers carrying 15 bases long 5' ends corresponding to the regions around EcoR1 site in the MCS of the bait vector. Then, the PCR was recombined with pGBKT7 linearized vector (by EcoR1 digestion) using the In-fusion HD cloning Kit (Clontech) according to the manufacturer 
protocol. The resulting recombinant plasmid pGBKT7-pVHL30 was sequenced by Sanger method and checked for protein expression by western blot.

\subsection{Library screening by yeast mating}

The yeast library screening was performed using the Mate \& Plate ${ }^{\mathrm{TM}}$ Library Human Testis (Clontech, Cat. $\mathrm{n}^{\circ}$ 630470). According to this, the library is expressed in Y187 yeast strain (MATa), conversely the bait protein was expressed in Y2HGold, a MAT $\alpha$ yeast strain. A fresh colony of a bait strain (Y2HGold [pGBKT7-pVHL30]) was inoculated in SD-Trp with shaking at $140 \mathrm{rpm}$ o/n at $30^{\circ} \mathrm{C}$. When the OD600 reached 0.8 , the culture was centrifugated and pellet re-suspended in $4 \mathrm{ml}$ of SD-Trp. In a sterile 3-L flask, $4 \mathrm{ml}$ of bait strain was combined with $1 \mathrm{ml}$ aliquot of testis library and $45 \mathrm{ml}$ of YPDA 2X liquid medium at $30{ }^{\circ} \mathrm{C}$ with slow shaking (around 30-50 rpm). After $30 \mathrm{~h}$ cells were centrifugated, pellet re-suspended in $10 \mathrm{ml}$ of 0,5X YPDA liquid medium and plated on $150 \mathrm{~mm}$ SD - Leu -Trp $+\mathrm{X}-\alpha-\mathrm{Gal}+\mathrm{AbA}\left(200 \mu \mathrm{l}\right.$ per plate). Plates were incubated at $30^{\circ} \mathrm{C}$ for 5 days monitoring colony growth twice a day. All positive clones were patched out on more stringency medium $\mathrm{QDO}+\mathrm{X}-\alpha-\mathrm{Gal}+\mathrm{AbA}$ in order to avoid false positives. The yeast host strains used were purchased from Clontech: Y2HGold (genotype: MATa, trp901, leu2-3, 112, ura3-52, his3-200, gal4 $\Delta$, gal80 $\Delta$, LYS2::GAL2UAS-Gal1TATAHIS3,GAL2UAS-Gal12TATA-Ade2, URA::MEL1UAS-Mel1TATA AUR1-c MEL1) and Y187 (genotype: MAT $\alpha$, ura3-52, his3-200, ade2-101, trp1-901, leu2-3, 112, gal4 $\Delta$, met-, gal80 $\Delta$, URA3 :: GAL1UAS-GAL1TATA-lacZ)

\subsection{Positive clones analysis}

DNA was extracted from yeast colonies using the Zymoprep ${ }^{\mathrm{TM}}-96$ Yeast Plasmid Miniprep (Zymo Research Cat. D2005) and $2 \mu \mathrm{l}$ of each DNA were used to transform TOP10 E. Coli (Calcium chloride competent cells homemade) according to standard protocol. 5 colonies of each transformation were analyzed. In this case, plasmidic DNAs were extracted using Zyppy TM-96 Plasmid Miniprep kit (Zymo Research Cat. D4041) and used as templates to amplify cDNA inserts with Gal4AD for and Gal4AD Rev primers. PCRs were performed according to Wondertaq protocol (Euroclone Cod. EME020001) and visualized on 0,8\% agarose gel. All positive clones with only one PCR product were transformed in Y190 yeast strain, provided by Euroscarf (genotype: MATa, gal4-542, gal80-538, his3, trp1-901, ade2-101, ura3-52, leu2-3, 112, URA3::GAL1-LacZ, Lys2::GAL1-HIS3cyhr). Each clone was co-transformed with pGBKT7 empty vector (as auto-activation control) and with pGBKT7-pVHL30 (as interaction control). For each transformation plate, two colonies were patched out on permissive medium (SD-Trp-Leu) and on selective ones (SD- Leu -Trp -His + 30mM or $60 \mathrm{mM} 3 \mathrm{AT}$ ). Finally, using the Gal4AD For primer, all positive clones were sequenced by the Sanger method.

\subsection{Positive clones identification and bioinformatic analysis}

To identify all the positive clones, nucleotide sequences were translated with Expasy Translate to retrieve the corresponding amino acid sequences. Resulting amino acid sequences were analyzed with BLASTP [27] against the UniProtKB database [28] (default options). Multiple sequence alignment to identify shared banding regions was performed with Jalview [29]. From a starting library including 142 clones, a total of 61 pVHL30 interactors were identified. The final dataset includes 6 pVHL30 interactors already described in the literature, while the remaining 55 are newly identified. Data about molecular function and subcellular localization for each interactor were retrieved from UniProtKB and Gene Ontology (GO) [30]. Protein-protein interaction network around the novel pVHL30 interactors was generated with Cytoscape [31], using molecular data from STRING [32] and BioGRID [33]. The preliminary network was extended with a second shell of STRING interactors retrieved setting parameters as follows: $\leq 10$ interactors, confidence $\geq 0.400$. MCODE [34] were used to identify clusters of highly interconnected proteins. Analysis of biochemical pathways, GO terms, and disease data was performed 
with Enrichr [35] and ClueGo [36]. Conservation of the core interaction networks across different tissues was investigated with HumanBase [37].

\section{Results}

\subsection{Yeast two-hybrid (Y2H) library screening in testis-tissue}

To investigate the pVHL30 interactome in testis-tissue we performed a large-scale screening using the yeast $S$. cerevisiae as organism model. The Y187 haploid (MATa) yeast strain expressing a human testis cDNA library (Clontech) was mated to the Y2HGold haploid strain (MAT $\alpha$ ) expressing pVHL30 as bait protein. To generate the bait, we cloned the cds of pVHL30 in pGBKT7 vector as described in Material and Methods section. The vector encodes the pVHL30 protein fused to the Gal4-DNA binding domain as verified by western blot (Supplementary Figure S1).

First, we checked for pVHL30 expression and its toxicity to exclude any alteration in yeast colonies growth-rate and their dimension. The growth of Y2HGold strain expressing pGBKT7VHL30 and prey empty vector was analyzed to exclude unspecific autoactivation and confirm that the yeast growth detected on selective media was dependent upon prey interaction. Mated culture was plated on selective media and plates incubated for 5 days at $30^{\circ} \mathrm{C}$ monitoring colonies number during the time. To reduce false positive, each positive clone identified after the mating was patched out on a more selective medium (Fig. 1). All these procedures allowed us to detect 607 positive clones. Total yeast DNA (i.e., genome, bait, and prey plasmids) was extracted from each of 607 yeast clone and analyzed by PCR using two primers able to pair vector sequences upstream and downstream the multiple cloning sites of the pGADT7 plasmid. Yeast clones characterized by the presence of multiple recombinant plasmids carrying different inserts were excluded from the identification process. All the other yeast DNAs were transformed in E. coli in order to amplify and isolate DNAs of prey plasmids. Finally, to further validate the pVHL30 interactors in a different genetic background each prey plasmid DNA was co-transformed with pGBKT7, empty or expressing pVHL30, in Y190 yeast strain and analyzed on selective media.

At the end of all these steps we detected 260 positive clones corresponding to an equal number of nucleotide sequences determined by Sanger sequencing.

A

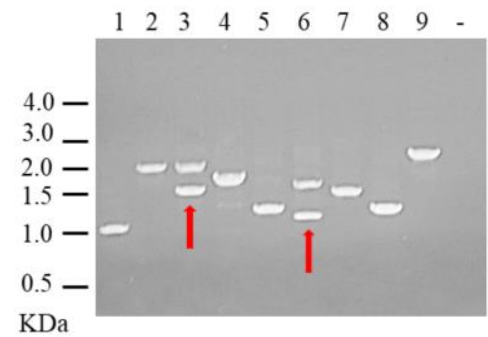

B

$\emptyset$ VHL30 $\varnothing$ VHL30

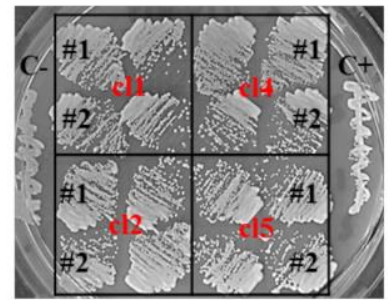

Permissive $\emptyset$ VHL30 $\varnothing$ VHL30

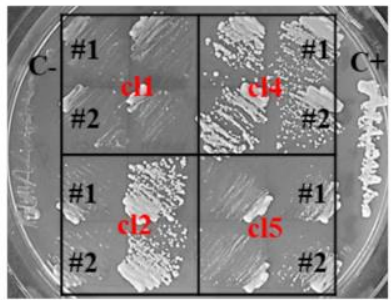

Selective $30 \mathrm{mM}$ 3-AT $\emptyset$ VHL30 $\varnothing$ VHL30

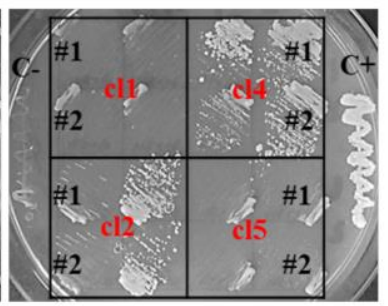

Selective $60 \mathrm{mM}$ 3-AT

Figure 1. Analysis of $\mathrm{Y} 2 \mathrm{H}$ library positive clones. A) PCR fragment visualization on agarose gel. Each line is marked with a number (n) corresponding to a library positive clone. The last column corresponds to the PCR negative control (-). Red arrows indicate clones with more than one insert, i.e., 3 and 6. B) Yeast patches. All positive clones were co-transformed with pGBKT7 empty (Ø) or pGBKT7 VHL30 (VHL30) as indicated on the top row. Two independent colonies (\#1, \#2) of each 
transformation were tested on permissive (right) and selective (left) media. C+ and C- correspond to $\mathrm{Y} 2 \mathrm{H}$ positive and negative control respectively.

\subsection{Identification and characterization of $p V H L 30$ interactors}

Resulting cDNA sequences were translated with ExPASy Translate and the amino acidic sequences used to perform BLAST search in UniProt. This step retrieved matches for 142 sequences. Sequences corresponding to putative pVHL30 binding motifs were identified from the library screening and mapped on the corresponding full-length protein to define the specific fragments involved (Table 1). Considering the presence of multiple clones encoding for a same protein, a total of 61 different pVHL30 interactors were identified from the library dataset. In detail, 6 proteins are already known pVHL interactors, whereas the remaining 55 candidates correspond to novel pVHL30 binding partners. According to VHLdb [18] the 6 known interactors are EEF1A1, CCT5, CCT7, Elongin-C, SNRNP200 and UBE2D2. Based on the relative clone frequencies, interactors were classified as $i$ ) highly represented characterized by a number of occurrences within 9 and 17, ii) average, between 4 and 6 each and iii) normal, with 3 occurrences each (Table 1 and Supplementary Table S1).

Table 1. List of proteins interacting with pVHL30. Known pVHL30 interactors are presented in grey.

\begin{tabular}{|c|c|c|c|}
\hline Protein name & UniProt ID & function & $\mathbf{n}^{\circ}$ hits \\
\hline Elongation factor 1-alpha 1 (EEF1A1) & P68104 & protein biosynthesis & 17 \\
\hline Elongin C (ELOC) & Q15369 & protein degradation & 6 \\
\hline T-complex protein 1 subunit eta (CCT7) & Q99832 & actin/tubulin folding & 4 \\
\hline Ubiquitin-coniugating enzyme E2 D2 (UBE2D2) & P62837 & protein ubiquitination & 2 \\
\hline T-complex protein 1 subunit epsilon (CCT5) & $\mathrm{P} 48643$ & actin/tubulin folding & 1 \\
\hline U5 small nuclear ribonucleoprotein 200 kDa helicase (SNRNP200) & O75643 & RNA splicing & 1 \\
\hline Putative methyltransferase (NSU7) & Q8NE18 & methylation & 12 \\
\hline Spermatogenesis-associated protein 22 (SPATA22) & Q8NHS9 & germ cell division & 10 \\
\hline Zinc finger and BTB domain-containing protein 17 (ZBTB17) & Q13105 & cell cycle regulator & 9 \\
\hline Microtubule-associated protein 1S (MAP1S) & Q66K74 & apoptosis & 4 \\
\hline Protein disulfide-isomerase A3 (PDIA3) & P30101 & protein folding & 4 \\
\hline Death-inducer obliterator 1 (DIDO1) & Q9BTC0 & tumor suppressor & 3 \\
\hline Electron transfer flavoprotein subunit alpha, mitochondrial (ETFA) & P13804 & electron transport & 3 \\
\hline 26S proteasome regulatory subunit 4 (PSMC1) & P62191 & protein degradation & 3 \\
\hline Histone deacetylase complex subunit (SAP30) & O75446 & deacetylation & 3 \\
\hline E3 ubiquitin-protein ligase (TTC3) & P53804 & $\begin{array}{c}\text { ubiquitination/protein } \\
\text { degradation }\end{array}$ & 3 \\
\hline Ankyrin repeat and EF-hand domain-containing protein 1 (ANKEF1) & Q9NU02 & n.d. & 2 \\
\hline Ankyrin repeat domain-containing protein 11 (ANKRD11) & Q6UB99 & chromatin regulator & 2 \\
\hline Guanylate kinase (GUK1) & B1ANH3 & phosphorylation & 2 \\
\hline Protein BEX4 (BEX4) & Q9NWD9 & $\begin{array}{l}\text { microtubule } \\
\text { deacetylation }\end{array}$ & 2 \\
\hline Cytochrome c oxidase subunit 2 (MT-CO2) & P00403 & oxygen reduction & 2 \\
\hline Prohibitin-2 (PHB2) & Q99623 & transcription inhibitor & 2 \\
\hline Piwi-like protein 4 (PIWIL4) & Q7Z3Z4 & tumor enhancer & 2 \\
\hline cGMP-dependent protein kinase 1 (PRKG1) & Q13976 & protein phosphorylation & 2 \\
\hline Arginine-glutamic acid dipeptide repeats protein (RERE) & Q9P2R6 & cell survival control & 2 \\
\hline Structural maintenance of chromosomes protein 5 (SMC5) & Q8IY18 & DNA repair & 2 \\
\hline STAM-binding protein (STAMBP) & O95630 & protein degradation & 2 \\
\hline
\end{tabular}




\begin{tabular}{|c|c|c|c|}
\hline Testis-expressed protein 35 (TEX35) & Q5T0J7 & n.d. & 2 \\
\hline Jouberin (AHI1) & Q8N157 & ciliogenesis & 1 \\
\hline Rho guanine nucleotide exchange factor 7 (ARHGEF7) & Q14155 & apoptosis & 1 \\
\hline Protein BEX2 (BEX2) & Q9BXY8-2 & cell cycle regulator & 1 \\
\hline Breast cancer type 1 susceptibility protein (BRCA1) & P38398 & E3-ub lig/DNA repair & 1 \\
\hline CDK5 regulatory subunit-associated protein 3 (CDK5RAP3) & J3QRX0 & n.d. & 1 \\
\hline COMM domain-containing protein 1 (COMMD1) & Q8N668 & $\begin{array}{l}\text { protein ubiquitination } \\
\text { regulator }\end{array}$ & 1 \\
\hline Copine-5 (CPNE5) & A0A0J9YWA1 & dendrite formation & 1 \\
\hline Destrin (DSTN) & P60981 & actin depolymerization & 1 \\
\hline 3'-5' exoribonuclease 1 (ERI1) & Q8IV48 & RNA exonuclease & 1 \\
\hline F-box only protein 28 (FBXO28) & Q9NVF7 & $\begin{array}{c}\text { ubiquitination/protein } \\
\text { degradation }\end{array}$ & 1 \\
\hline F-box only protein 34 (FBXO34) & Q9NWN3 & SRP of E3-ub complex & 1 \\
\hline Flotillin-1 (FLOT1) & O75955 & caveolae formation & 1 \\
\hline G patch domain and ankyrin repeat-containing protein 1 (GPANK1) & O95872 & n.d. & 1 \\
\hline General transcription factor 3C polypeptide 2 (GTF3C2) & Q8WUA4 & DNA transcription & 1 \\
\hline Intraflagellar transport protein 88 homolog (IFT88) & Q13099 & ciliogenesis & 1 \\
\hline Inositol-trisphosphate 3-kinase (ITPKC) & Q96DU7 & phosphorylation & 1 \\
\hline Kelch-like protein 10 (KLHL10) & Q6JEL2 & $\begin{array}{c}\text { ubiquitination/protein } \\
\text { degradation }\end{array}$ & 1 \\
\hline Microtubule-associated protein 1B (MAP1B) & P46821 & microtubule stabilization & 1 \\
\hline $28 \mathrm{~S}$ ribosomal protein S9, mitochondrial (MRPS9) & P82933 & n.d. & 1 \\
\hline E3 ubiquitin-protein ligase MSL2 (MSL2) & Q9HCI7 & $\begin{array}{c}\text { ubiquitination/protein } \\
\text { degradation }\end{array}$ & 1 \\
\hline Nischarin (NISCH) & Q9Y2I1 & cell survival/migration & 1 \\
\hline Probable 28S rRNA (cytosine-C(5))-methyltransferase (NSUN5) & Q96P11 & methylation & 1 \\
\hline ATP-dependent 6-phosphofructokinase, platelet type (PFKP) & Q01813 & glycolysis & 1 \\
\hline Plakophilin-2 (PKP2) & Q99959 & cell-cell adhesion & 1 \\
\hline Selenoprotein P (SELENOP) & P49908 & selenium transport & 1 \\
\hline SPARC & P09486 & cell growth & 1 \\
\hline Spermatogenic leucine zipper protein 1 (SPZ1) & Q9BXG8 & $\begin{array}{l}\text { germ cell proliferation } \\
\text { and differentiation }\end{array}$ & 1 \\
\hline Histone-lysine N-methyltransferase (SUV39H2) & Q9H5I1 & chromatin regulator & 1 \\
\hline Tudor domain-containing protein 7 (TDRD7) & Q8NHU6 & $\begin{array}{c}\text { post-transcription } \\
\text { regulator }\end{array}$ & 1 \\
\hline Zinc finger protein 200 (ZNF200) & P98182 & spermatogenesis & 1 \\
\hline Zinc finger protein 668 (ZNF668) & Q96K58 & transcription regulator & 1 \\
\hline Zinc finger protein 827 (ZNF827) & Q17R98 & transcription regulator & 1 \\
\hline
\end{tabular}

We then wondered whether fragments belonging to a same protein could be used to refine identification of pVHL binding motifs. To this aim, we extracted candidate interactors presenting more than 2 occurrences and aligned their sequences to identify shared regions - if any - among different clones. As an example of the analysis workflow, we report the alignment generated using fragments belonging from the Elongation factor 1-alpha 1 (EEF1A1), the most represented protein in our dataset (Fig. 2). 
Figure 2. EEF1A1 multiple sequence alignment. Amino acid sequences corresponding to EEF1A1 (UniProt_ID P68104) derived from different clones. The region shared among all clones highlights the putative pVHL30 binding motifs.

In particular, we identified 17 hits corresponding to 12 different amino acid sequences. These were aligned and compared with the EEF1A1 reference sequence retrieved by UniProt [28] (ID P68104). We found a fragment of 27 residues (aa 297- 324) shared among all clones (Fig. 2), suggesting this region to be the pVHL30 binding motif. Following this approach for each interactor, a total of 12 different promising pVHL30 binding fragments were identified (Supplementary Table S1). In our library screening we also identified multiple fragments from the same protein sharing no significant sequence identity suggesting that each of them possesses unique features. These fragments were then investigated for secondary structure content, presence of functional domains, functional linear motifs and post-translational modification sites. Interestingly, by searching against the ELM database [38] we found presence of a so-called "USP7-binding motif" in three proteins, i.e., NOP2/Sun RNA Methyltransferase Family Member 7 (NSUN7), Spermatogenesis Associated 22 (SPATA22) and Protein Disulfide Isomerase Family A Member 3 (PDIA3). The Ubiquitin Specific Peptidase 7 (USP7) is a deubiquitinating enzyme involved in p53 stabilization [39] presenting high affinity for MDM2, the main E3 ubiquitin-ligase involved in p53 degradation [40]. The pVHL30 was recently proposed to bind MDM2 up-stream to the USP7 interacting motif [41]. Moreover, pVHL30 is also known to interact with both USP33 and USP20, two enzymes belonging to the USP protein family, whose USP7 is a further member [42]. Data from the literature indicate that pVHL30 mediates the ubiquitination and degradation of both USP33 and USP20, in turn down-regulating the pathways controlled by these two proteins [43]. These findings may suggest that pVHL30 plays a role in regulating MDM2/USP7 association.

\subsection{Sub-cellular localization of the new identified pVHL30 interactors.}

The new pVHL30 binding partners were found to preferentially localize into the nucleus (29 interactors), while 7 are cytosolic proteins. The remaining identified interactors are almost equally distributed among endosome, membrane, chromosome, and endoplasmic reticulum. Only one protein was found to participate in the lysosome, i.e., cathepsin D (CTSD), whereas no interactors residing into the Golgi were found. Interestingly, among the novel putative pVHL30 interactors, we also identified four mitochondrial proteins, i.e., ETFA, MRPS9, MTCO2 and PHB2, mainly involved in the regulation of mitochondrial respiration activity and electron transfer to the mitochondrial respiratory chain. The predominance of nuclear proteins is not surprising per se as the nucleus represents one of the subcellular compartments in which pVHL is normally found at physiological conditions. Furthermore, the pVHL sub-localization is thought to be regulated in a cell cycle-dependent manner that influences the protein shuttling between the nucleus and the cytoplasm [44]. The pVHL30 association with CTSD is, nevertheless, particularly interesting. A recent work demonstrated a lysosomal vulnerability in VHLinactivated RCC cells, at least in vitro [45]. In other words, renal cancer cells possessing unfunctional pVHL seem unable to maintain their lysosomes localization upon drug- 
induced stress. We propose that the association identified here between pVHL30 and CTSD may play a role in renal cancer progression.

\subsection{Prediction of $p V H L 30$ binding motifs}

We then investigated our library of fragments with DiLiMOT [46] to identify cryptic pVHL30 binding motifs. According to the significance threshold, six motifs were marked as good candidates (Table 2). The two most represented are VGxxxK and PxxxVxxN, each shared among 4 different pVHL30 interactors. Further, both these putative motifs are present in the EEF1A1 that is a well-known pVHL30 binder [47]. This evidence supports their possible involvement in pVHL30 binding. The remaining proposed motifs are rich in lysines (i.e., GxxKxxK, KKKxK, KxKxKxK, KxxxPK). Lysine is a positively charged amino acid as well as it is a target site of post-translational modifications. According to their electrostatic net charge, these motifs could likely mediate binding with the pVHL30 $\mathrm{N}$-terminal tail, which is characterized by a strong concentration of negatively charged residues [8]. The possibility to turn off the pVHL30 interaction with these motifs by lysine methylation is fascinating. It should be mentioned that these last 4 motifs were predicted with a P-value below the significance threshold, however it doesn't completely exclude their reliability, as real binder motifs can still occur also with a less significant value.

Table 2. Putative pVHL30 interacting motifs identified by DiLiMOT.

\begin{tabular}{cccc}
\hline Motif & Scons & $\mathbf{N}^{\circ}$ protein & P value \\
\hline VGxxxK & $2.22 \mathrm{e}-29$ & 4 & $2.84 \mathrm{e}-05$ \\
\hline PxxxVxxN & $3.12 \mathrm{e}-24$ & 4 & $2.20 \mathrm{e}-05$ \\
\hline GxKxxK & $1.42 \mathrm{e}-22$ & 4 & $2.48 \mathrm{e}-04$ \\
\hline KKKxK & $1.13 \mathrm{e}-20$ & 4 & $9.00 \mathrm{e}-06$ \\
\hline KxKxKxK & $1.39 \mathrm{e}-18$ & 4 & $3.81 \mathrm{e}-06$ \\
\hline KxxxPK & $3.10 \mathrm{e}-18$ & 5 & $1.29 \mathrm{e}-05$ \\
\hline KxxKxxxP & $1.20 \mathrm{e}-17$ & 4 & $2.37 \mathrm{e}-04$ \\
\hline KNxxxK & $1.63 \mathrm{e}-16$ & 4 & $3.15 \mathrm{e}-04$ \\
\hline AxxVP & $3.45 \mathrm{e}-16$ & 4 & $2.18 \mathrm{e}-04$ \\
\hline KKK & $4.05 \mathrm{e}-16$ & 5 & $2.76 \mathrm{e}-04$ \\
\hline
\end{tabular}

\subsection{Mutations found in cancer affect the $p$ VHL30 binding motifs}

We decided then to verify whether cancer-associated mutations localize within the pVHL binding fragments. Search against COSMIC [48] identified a number of mutations found in tumors (i.e. adenocarcinoma, squamous cell carcinoma, malignant melanoma and clear cell renal cell carcinoma) localizing within fragments corresponding to SPATA22, ZBTB17, CCT7, MAP1S and PDIA3 proteins. In particular, missense mutations such as the p.Arg89Ile in SPATA22, p.Arg562Cys and p.Arg625Trp in ZBTB17, p.Pro235Ser and p.Glu316Lys in CCT7, p.Arg863Gln and p.Gly891Ser in MAP1S as well as synonymous substitutions pSer497Ser in ZBTB17 and p.Leu361Leu in PDIA3 seem to correlate with pathogenic conditions (Table 3). Considering the aforementioned findings, it can be speculated that mutations in these positions can alter the pVHL30 binding affinity thus conferring pro-oncogenic features to cells harboring them. Further investigations are however required to address this evidence.

Table 3. New pVHL interactors found mutated in cancers and related amino acid variants. 


\begin{tabular}{ccc} 
SPATA 22 & p.Arg89le & glioma and adenocarcinoma (large intestine) \\
\hline \multirow{2}{*}{ ZBTB17 } & p.Arg562Cys & carcinoma (endometrium, thyroid) \\
& $\begin{array}{c}\text { p.Arg625Trp } \\
\text { p.Ser497= }\end{array}$ & $\begin{array}{c}\text { carcinoma (large cell) } \\
\text { CCT7 }\end{array}$ \\
& p.Pro235Ser & adenoma (large intestine) and carcinoma (upper aerodigestive tract) \\
\hline \multirow{2}{*}{ p.Glu316Lys } & malignant melanoma \\
& p.Arg863Asn & adenocarcinoma (lung, urinary tract) \\
\hline \multirow{2}{*}{ PDIS } & p.Gly891Ser & adenocarcinoma (large intestine) \\
& p.Leu361= & carcinoma (urinary tract) \\
\hline
\end{tabular}

\subsection{Pathway analysis of $p V H L 30$ binding motifs}

To further investigate the pathways in which these novel interactors are involved, a protein interacting network centered around pVHL30 was created using STRING [32]. Resulting network shows no trivial interaction among the 61 proteins except for 5 entries which form a small cluster. For this reason, the preliminary interacting network was enriched by including a second layer of interactors (setting "no more than 50 proteins" for each interactor). The extended network shows pVHL30 partners to be associated with multiple relevant cellular pathways (Fig. 3). In particular, a major central cluster composed of 43 proteins highly interconnected was identified. Among them, PSMC1, GUK1 and STAMBP1, EEF1A1, CCT5 and CCT7 are pVHL30 partners identified by the $\mathrm{Y} 2 \mathrm{H}$ screening. This cluster seems to have a bi-modal distribution with a most populated fraction containing HIF- $1 \alpha$ and several proteins involved in protein degradation. This finding is expected as it is confirmatory of the known pVHL30 involvement in this process. This portion of the cluster also includes PSMC1 and multiple proteins forming the proteasome subunits. The proteasome plays a key role in the maintenance of protein homeostasis by removing misfolded or damaged proteins, which could impair cellular functions, and by removing proteins whose functions are no longer required [49]. It participates in numerous cellular processes, including cell cycle progression, apoptosis, or DNA damage repair, processes in which pVHL30 is also involved. On the other hand, in the same cluster were also found several proteins belonging from the chaperonin family (e.g., CCT5, CCT7) known to play a key role in the correct folding of cytoskeleton proteins. This evidence can be related to the pVHL30 involvement in the regulation of microtubule dynamics [50], an example of HIF- $1 \alpha$ independent pVHL function. A second interesting cluster includes DIDO1, GTF3C2, SNRNP200 and PFKP. This cluster is composed of 11 nodes and centered around CDC5L, a protein with a key role in cell-cycle regulation and spliceosome activation [51]. Pathway analysis showed that clusters formed by the novel pVHL30 interactors are enriched in multiple biological processes such as regulation of epithelial cell differentiation, synaptic plasticity, histone methylation, chromatin disassembly and negative regulation of microtubule nucleation. 
Collectively, these findings highlight already known pVHL30 functions. Moreover, a number of interactors identified in our screening showed no trivial interconnections suggesting that pVHL30 may directly link these proteins, therefore putatively also exerting novel not yet characterized functions.
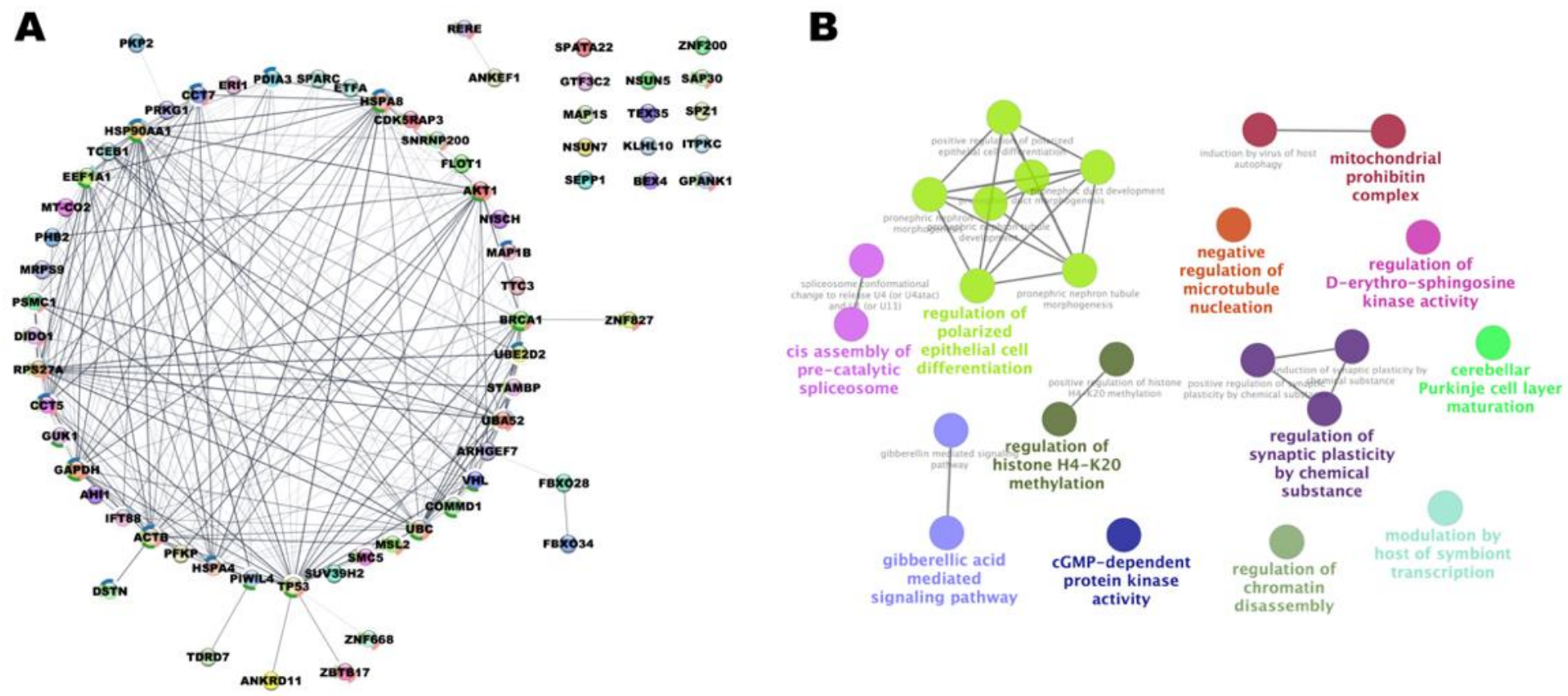

Figure 3. Protein-protein interaction network of novel testis-derived pVHL30 interactors. A) interaction network presented as circular layout, with bubbles representing pVHL30 interactors while edges are functional connection reported in the literature. Edge coloring intensity reflects the number of different evidence connecting nodes. A bubble is rounded by a colored circle when the node is shared among different pathways. B) different biological function associated with clusters of pVHL30 interactors $(\mathrm{P}<0.05)$.

\section{7. pVHL30 interaction with 3'- or 5'-untraslated regions (UTR)}

In our library screening we also identified other 86 entries that correspond to peptides containing non-coding region, i.e., 3'- or 5'-untraslated regions (UTR). Together, the $\mathrm{Y} 2 \mathrm{H}$ library screening produced two different datasets, one accounting for 142 entries corresponding to 61 different proteins and a second dataset collecting other 86 pVHL30 interacting amino acid sequences not corresponding to individual coding regions. It is known in the literature that $\mathrm{Y} 2 \mathrm{H}$ screening results in identification of many out-of-frame (OOF) clones that encode peptides with no sequence homology to known proteins [52]. These OOF clones typically generate during library construction from cloning restrictiondigested cDNAs fused to the AD. Nevertheless, deeper analysis of these peptides could still reveal common short linear motifs (SLiMs) responsible for their selection. In other words, they can represent a further reservoir of putative new linear motifs able to mediate association with pVHL30 (Supplementary Table S2).

\section{Discussion}

The $\mathrm{Y} 2 \mathrm{H}$ screening is a useful technique vastly used to construct extensive proteinprotein interaction maps for humans and several model organisms [53]. In this study, we adopted this approach to investigate the interactome around the human pVHL30 in testis. The analysis identified 260 positive clones that were further classified into two main 
groups: 61 human proteins and 86 peptides derived from noncoding regions. We found six known pVHL30 interactors that were used to confirm the screening system reliability. Indeed, pVHL30 was correctly detected as binder of Elongin C, EEF1A1, CCT5, CCT7, SNRNP200 and UBE2D2 interactors, suggesting the general "good ability" of this technique to find out in vivo real associations outside the physiological context in which they normally occur. On the other hand, this approach allowed us to also identify 55 novel pVHL30 interactors, including proteins involved in relevant cellular pathways, such as the cell-cycle regulation, DNA damage repair, apoptosis, and cytoskeleton regulation. Interestingly, in multiple cases our analysis suggested specific pVHL30 binding motifs which represent a valuable starting point for the identification of functional linear motifs. As expected, our screening highlighted novel tissue-specific interaction of pVHL30 with proteins relevant for the correct testis function. Among them, we found proteins regulating spermatogenesis, such as the Piwi-like protein 4 (PIWIL4), Spermatogenic leucine zipper protein 1 (SPZ1) and Spermatogenesis-associated protein 22 (SPATA22), indicating that pVHL30 could be functionally linked to sperm-cell proliferation and differentiation also in human. The PIWIL4 is known to represses transposable elements and prevents their mobilization during spermatogenesis ensuring germline integrity [54]. This protein also plays crucial roles in somatic cells, such as participating in the correct maintenance of the retinal epithelial through Akt/GSK3alpha/beta signaling axis [55] and regulating the pancreatic beta cells function [56]. Both these tissues are target sites for cancer insurgence in VHL syndrome, whose affected patients develop retinal hemangioblastomas and pancreatic cystadenomas. A possible role for the interaction between pVHL30 and PIWIL4 in the formation of these two cancers is at least fascinating and will require further investigations. We also found interactions with multiple proteins regulating metabolism, gene expression and cell cycle progression. These findings further support an implication of pVHL30 in sperm cells maturation. In this sense, its association with Prohibitin-2 is of interest. In mitochondria, Prohibitin-2 is a chaperone-like acting protein involved in the stabilization of mitochondrial respiratory enzymes [57], a role which is critical for sperm cell development. Indeed, to allow sperm survival during its scrotal storage, the balance between oxidative metabolism and redox homeostasis has to be finely tuned and sophisticated regulatory mechanisms were evolved by nature [58]. Considered the main pVHL30 function in mediating the hypoxia response, the interaction with Prohibitin-2 opens possible new biological roles for this protein. As an example, epididymal cystadenoma (ECs) is frequently found in association with VHL disease, however only little is known about the specific pVHL30 functions in this tissue. Our findings could help to shed new light on this type of cancer. Similarly, we also found interaction with CTSD a lysosomal aspartyl protease proposed as a relevant diagnostic and prognostic biomarker for RCC [59]. Indeed, increased excretion of CTSD in RCC is not consequence of its increased transcription [59], rather it is thought to be due to protein relocation or other mechanisms. The biological meaning behind CTSD association with pVHL30 is currently unknown, however, based on our findings we speculate that pVHL30 may be involved in the degradation of CTSD as VHL gene is lost or unfunctional in over $70 \%$ of sporadic RCC $[60,61]$. Another interesting point to discuss, is the ability of pVHL30 to recognize and interact with the UTR regions, as showed by the interaction with 86 entries codifying for out-of-frames clones. The UTRs regions are known to have key roles in post-transcriptional gene regulation for the maintenance of cellular homeostasis as they contain different regulatory elements involved in pre-mRNA processing, mRNA stability and translation initiation [62]. In a contest of cancer-induced genetic instability, it can be proposed that these UTRs may fuse with regular codifying regions yielding chimeric protein products able to associate pVHL30. Thus, it can be of interest to investigate whether this specific gene rearrangement happens in cancer cells and how it impacts pVHL30 functions.

Collectively, our library screening showed that pVHL30 can interact with multiple new proteins suggesting novel testis-specific functions. Further investigations for each 
new pVHL30 interactor will be necessary to understand the functional meaning beyond these binary associations.

\title{
5. Conclusions
}

We described the testis-specific proteome around the human pVHL30 obtained by library screening. Our approach identified 55 novel pVHL30 interactors, with multiple proteins directly involved in spermatogenesis, reproductive metabolism, and cancer. Albeit further study is warranted to elucidate the exact role of these new interactions, we demonstrated that pVHL30 can bind tissue-specific interactors and suggested novel roles for this oncosuppressor protein.

\begin{abstract}
Author Contributions: G.M. and S.T. Conceptualization; A.F., G.M. and G. S. methodology; A.F. and G.M. investigation; A.F., and G.M. formal analysis and validation; F.Q., and G.M. data curation and bioinformatics analysis; A.F., G.M., S.T. original draft preparation and editing. All authors have read and agreed to the published version of the manuscript.
\end{abstract}

Funding: This research was funded by Fondazione AIRC per la Ricerca sul Cancro (AIRC), grant number IG 2019 ID. 23825.

Acknowledgments: We acknowledge Dr. Raffaele Lopreiato for the helpful discussions.

Conflicts of Interest: The authors declare no conflict of interest. The funders had no role in the design of the study; in the collection, analyses, or interpretation of data; in the writing of the manuscript, or in the decision to publish the results.

\section{References}

1. Shuin, T.; Yamasaki, I.; Tamura, K.; Okuda, H.; Furihata, M.; Ashida, S. Von Hippel-Lindau Disease: Molecular Pathological Basis, Clinical Criteria, Genetic Testing, Clinical Features of Tumors and Treatment. Jpn J Clin Oncol 2006, 36, 337-343, doi:10.1093/jjco/hyl052.

2. Nordstrom-O’Brien, M.; van der Luijt, R.B.; van Rooijen, E.; van den Ouweland, A.M.; MajoorKrakauer, D.F.; Lolkema, M.P.; van Brussel, A.; Voest, E.E.; Giles, R.H. Genetic Analysis of von HippelLindau Disease. Hum. Mutat. 2010, 31, 521-537, doi:10.1002/humu.21219.

3. $\quad$ Crespigio, J.; Berbel, L.C.L.; Dias, M.A.; Berbel, R.F.; Pereira, S.S.; Pignatelli, D.; Mazzuco, T.L. Von Hippel-Lindau Disease: A Single Gene, Several Hereditary Tumors. J Endocrinol Invest 2017, 1-11, doi:10.1007/s40618-017-0683-1.

4. $\quad$ Latif, F.; Tory, K.; Gnarra, J.; Yao, M.; Duh, F.M.; Orcutt, M.L.; Stackhouse, T.; Kuzmin, I.; Modi, W.; Geil, L. Identification of the von Hippel-Lindau Disease Tumor Suppressor Gene. Science 1993, 260, 1317-1320.

5. Iliopoulos, O.; Ohh, M.; Kaelin, W.G. PVHL19 Is a Biologically Active Product of the von HippelLindau Gene Arising from Internal Translation Initiation. Proc. Natl. Acad. Sci. U.S.A. 1998, 95, 1166111666.

6. Chesnel, F.; Hascoet, P.; Gagné, J.P.; Couturier, A.; Jouan, F.; Poirier, G.G.; Le Goff, C.; Vigneau, C.; Danger, Y.; Verite, F.; et al. The von Hippel-Lindau Tumour Suppressor Gene: Uncovering the Expression of the PVHL172 Isoform. British Journal of Cancer 2015, 113, 336-344, doi:10.1038/bjc.2015.189.

7. Min, J.-H.; Yang, H.; Ivan, M.; Gertler, F.; Kaelin, W.G.; Pavletich, N.P. Structure of an HIF-1alpha -PVHL Complex: Hydroxyproline Recognition in Signaling. Science 2002, 296, 1886-1889, doi:10.1126/science.1073440.

8. Minervini, G.; Mazzotta, G.M.; Masiero, A.; Sartori, E.; Corrà, S.; Potenza, E.; Costa, R.; Tosatto, S.C.E. Isoform-Specific Interactions of the von Hippel-Lindau Tumor Suppressor Protein. Sci Rep 2015, 5, 12605, doi:10.1038/srep12605.

9. $\quad$ Kamura, T.; Koepp, D.M.; Conrad, M.N.; Skowyra, D.; Moreland, R.J.; Iliopoulos, O.; Lane, W.S.; Kaelin, W.G.; Elledge, S.J.; Conaway, R.C.; et al. Rbx1, a Component of the VHL Tumor Suppressor Complex and SCF Ubiquitin Ligase. Science 1999, 284, 657-661, doi:10.1126/science.284.5414.657. 10. Stebbins, C.E.; Kaelin, W.G.; Pavletich, N.P. Structure of the VHL-ElonginC-ElonginB Complex: 
Implications for VHL Tumor Suppressor Function. Science 1999, 284, 455-461, doi:10.1126/science.284.5413.455.

11. Epstein, A.C.R.; Gleadle, J.M.; McNeill, L.A.; Hewitson, K.S.; O’Rourke, J.; Mole, D.R.; Mukherji, M.; Metzen, E.; Wilson, M.I.; Dhanda, A.; et al. C. Elegans EGL-9 and Mammalian Homologs Define a Family of Dioxygenases That Regulate HIF by Prolyl Hydroxylation. Cell 2001, 107, 43-54, doi:10.1016/S0092-8674(01)00507-4.

12. Ohh, M.; Yauch, R.L.; Lonergan, K.M.; Whaley, J.M.; Stemmer-Rachamimov, A.O.; Louis, D.N.; Gavin, B.J.; Kley, N.; Kaelin Jr., W.G.; Iliopoulos, O. The von Hippel-Lindau Tumor Suppressor Protein Is Required for Proper Assembly of an Extracellular Fibronectin Matrix. Molecular Cell 1998, 1, 959-968, doi:10.1016/S1097-2765(00)80096-9.

13. Young, A.P.; Schlisio, S.; Minamishima, Y.A.; Zhang, Q.; Li, L.; Grisanzio, C.; Signoretti, S.; Kaelin, W.G. VHL Loss Actuates a HIF-Independent Senescence Programme Mediated by Rb and P400. Nat. Cell Biol. 2008, 10, 361-369, doi:10.1038/ncb1699.

14. Guo, J.; Chakraborty, A.A.; Liu, P.; Gan, W.; Zheng, X.; Inuzuka, H.; Wang, B.; Zhang, J.; Zhang, L.; Yuan, M.; et al. PVHL Suppresses Kinase Activity of Akt in a Proline-Hydroxylation-Dependent Manner. Science 2016, 353, 929-932, doi:10.1126/science.aad5755.

15. Minervini, G.; Pennuto, M.; Tosatto, S.C.E. The PVHL Neglected Functions, a Tale of HypoxiaDependent and -Independent Regulations in Cancer. Open Biology 10, 200109, doi:10.1098/rsob.200109. 16. Roe, J.-S.; Youn, H.-D. The Positive Regulation of P53 by the Tumor Suppressor VHL. Cell Cycle 2006, 5, 2054-2056, doi:10.4161/cc.5.18.3247.

17. Kerrien, S.; Aranda, B.; Breuza, L.; Bridge, A.; Broackes-Carter, F.; Chen, C.; Duesbury, M.; Dumousseau, M.; Feuermann, M.; Hinz, U.; et al. The IntAct Molecular Interaction Database in 2012. Nucleic Acids Res. 2012, 40, D841-846, doi:10.1093/nar/gkr1088.

18. Tabaro, F.; Minervini, G.; Sundus, F.; Quaglia, F.; Leonardi, E.; Piovesan, D.; Tosatto, S.C.E. VHLdb: A Database of von Hippel-Lindau Protein Interactors and Mutations. Scientific Reports 2016, 6, 31128, doi:10.1038/srep31128.

19. Ma, W.; Tessarollo, L.; Hong, S.-B.; Baba, M.; Southon, E.; Back, T.C.; Spence, S.; Lobe, C.G.; Sharma, N.; Maher, G.W.; et al. Hepatic Vascular Tumors, Angiectasis in Multiple Organs, and Impaired Spermatogenesis in Mice with Conditional Inactivation of the VHL Gene. Cancer Res 2003, 63, 5320-5328. 20. Choyke, P.L.; Glenn, G.M.; Wagner, J.P.; Lubensky, I.A.; Thakore, K.; Zbar, B.; Linehan, W.M.; Walther, M.M. Epididymal Cystadenomas in von Hippel-Lindau Disease. Urology 1997, 49, 926-931, doi:10.1016/s0090-4295(97)00074-5.

21. Papadakis, G.Z.; Millo, C.; Sadowski, S.M.; Bagci, U.; Patronas, N.J. Epididymal Cystadenomas in von Hippel-Lindau Disease Showing Increased Activity on 68Ga-DOTA-TATE PET/CT. Clin Nucl Med 2016, 41, 781-782, doi:10.1097/RLU.0000000000001314.

22. Cox, R.; Vang, R.; Epstein, J.I. Papillary Cystadenoma of the Epididymis and Broad Ligament: Morphologic and Immunohistochemical Overlap With Clear Cell Papillary Renal Cell Carcinoma. The American Journal of Surgical Pathology 2014, 38, 713-718, doi:10.1097/PAS.0000000000000152.

23. Schermer, B.; Ghenoiu, C.; Bartram, M.; Müller, R.U.; Kotsis, F.; Höhne, M.; Kühn, W.; Rapka, M.; Nitschke, R.; Zentgraf, H.; et al. The von Hippel-Lindau Tumor Suppressor Protein Controls Ciliogenesis by Orienting Microtubule Growth. J Cell Biol 2006, 175, 547-554, doi:10.1083/jcb.200605092.

24. Girardet, L.; Augière, C.; Asselin, M.-P.; Belleannée, C. Primary Cilia: Biosensors of the Male Reproductive Tract. Andrology 2019, 7, 588-602, doi:10.1111/andr.12650.

25. Higgins, M.; Obaidi, I.; McMorrow, T. Primary Cilia and Their Role in Cancer (Review). Oncology Letters 2019, 17, 3041-3047, doi:10.3892/ol.2019.9942.

26. Adamiok-Ostrowska, A.; Piekiełko-Witkowska, A. Ciliary Genes in Renal Cystic Diseases. Cells 2020, 9, E907, doi:10.3390/cells9040907.

27. States, D.J.; Gish, W. Combined Use of Sequence Similarity and Codon Bias for Coding Region Identification. J Comput Biol 1994, 1, 39-50, doi:10.1089/cmb.1994.1.39.

28. The UniProt Consortium UniProt: A Worldwide Hub of Protein Knowledge. Nucleic Acids Research 2019, 47, D506-D515, doi:10.1093/nar/gky1049. 
29. Waterhouse, A.M.; Procter, J.B.; Martin, D.M.A.; Clamp, M.; Barton, G.J. Jalview Version 2--a Multiple Sequence Alignment Editor and Analysis Workbench. Bioinformatics 2009, 25, 1189-1191, doi:10.1093/bioinformatics/btp033.

30. Gene Ontology Consortium The Gene Ontology Resource: Enriching a GOld Mine. Nucleic Acids Res 2021, 49, D325-D334, doi:10.1093/nar/gkaa1113.

31. Shannon, P.; Markiel, A.; Ozier, O.; Baliga, N.S.; Wang, J.T.; Ramage, D.; Amin, N.; Schwikowski, B.; Ideker, T. Cytoscape: A Software Environment for Integrated Models of Biomolecular Interaction Networks. Genome Res. 2003, 13, 2498-2504, doi:10.1101/gr.1239303.

32. $\quad$ Szklarczyk, D.; Gable, A.L.; Nastou, K.C.; Lyon, D.; Kirsch, R.; Pyysalo, S.; Doncheva, N.T.; Legeay, M.; Fang, T.; Bork, P.; et al. The STRING Database in 2021: Customizable Protein-Protein Networks, and Functional Characterization of User-Uploaded Gene/Measurement Sets. Nucleic Acids Res 2021, 49, D605-D612, doi:10.1093/nar/gkaa1074.

33. Chatr-aryamontri, A.; Oughtred, R.; Boucher, L.; Rust, J.; Chang, C.; Kolas, N.K.; O’Donnell, L.; Oster, S.; Theesfeld, C.; Sellam, A.; et al. The BioGRID Interaction Database: 2017 Update. Nucleic Acids Research 2016, doi:10.1093/nar/gkw1102.

34. Bader, G.D.; Hogue, C.W. An Automated Method for Finding Molecular Complexes in Large Protein Interaction Networks. BMC Bioinformatics 2003, 4, 2, doi:10.1186/1471-2105-4-2.

35. Kuleshov, M.V.; Jones, M.R.; Rouillard, A.D.; Fernandez, N.F.; Duan, Q.; Wang, Z.; Koplev, S.; Jenkins, S.L.; Jagodnik, K.M.; Lachmann, A.; et al. Enrichr: A Comprehensive Gene Set Enrichment Analysis Web Server 2016 Update. Nucleic Acids Res 2016, 44, W90-W97, doi:10.1093/nar/gkw377.

36. Bindea, G.; Mlecnik, B.; Hackl, H.; Charoentong, P.; Tosolini, M.; Kirilovsky, A.; Fridman, W.-H.; Pagès, F.; Trajanoski, Z.; Galon, J. ClueGO: A Cytoscape Plug-in to Decipher Functionally Grouped Gene Ontology and Pathway Annotation Networks. Bioinformatics 2009, 25, 1091-1093, doi:10.1093/bioinformatics/btp101.

37. Greene, C.S.; Krishnan, A.; Wong, A.K.; Ricciotti, E.; Zelaya, R.A.; Himmelstein, D.S.; Zhang, R.; Hartmann, B.M.; Zaslavsky, E.; Sealfon, S.C.; et al. Understanding Multicellular Function and Disease with Human Tissue-Specific Networks. Nat Genet 2015, 47, 569-576, doi:10.1038/ng.3259.

38. Kumar, M.; Gouw, M.; Michael, S.; Sámano-Sánchez, H.; Pancsa, R.; Glavina, J.; Diakogianni, A.; Valverde, J.A.; Bukirova, D.; Čalyševa, J.; et al. ELM - the Eukaryotic Linear Motif Resource in 2020. Nucleic Acids Res 2020, 48, D296-D306, doi:10.1093/nar/gkz1030.

39. Li, M.; Chen, D.; Shiloh, A.; Luo, J.; Nikolaev, A.Y.; Qin, J.; Gu, W. Deubiquitination of P53 by HAUSP Is an Important Pathway for P53 Stabilization. Nature 2002, 416, 648-653, doi:10.1038/nature737. 40. Haupt, Y.; Maya, R.; Kazaz, A.; Oren, M. Mdm2 Promotes the Rapid Degradation of P53. Nature 1997, 387, 296-299, doi:10.1038/387296a0.

41. Falconieri, A.; Minervini, G.; Bortolotto, R.; Piovesan, D.; Lopreiato, R.; Sartori, G.; Pennuto, M.; Tosatto, S.C.E. The E3 Ubiquitin-Protein Ligase MDM2 Is a Novel Interactor of the von Hippel-Lindau Tumor Suppressor. Sci Rep 2020, 10, 15850, doi:10.1038/s41598-020-72683-3.

42. $\quad$ Li, Z.; Wang, D.; Messing, E.M.; Wu, G. VHL Protein-Interacting Deubiquitinating Enzyme 2 Deubiquitinates and Stabilizes HIF-1alpha. EMBO Rep 2005, 6, 373-378, doi:10.1038/sj.embor.7400377. 43. Li, Z.; Wang, D.; Na, X.; Schoen, S.R.; Messing, E.M.; Wu, G. Identification of a Deubiquitinating Enzyme Subfamily as Substrates of the von Hippel-Lindau Tumor Suppressor. Biochem Biophys Res Commun 2002, 294, 700-709, doi:10.1016/S0006-291X(02)00534-X.

44. Ye, Y.; Vasavada, S.; Kuzmin, I.; Stackhouse, T.; Zbar, B.; Williams, B.R. Subcellular Localization of the von Hippel-Lindau Disease Gene Product Is Cell Cycle-Dependent. Int J Cancer 1998, 78, 62-69, doi:10.1002/(sici)1097-0215(19980925)78:1<62::aid-ijc11>3.0.co;2-7.

45. Bouhamdani, N.; Comeau, D.; Coholan, A.; Cormier, K.; Turcotte, S. Targeting Lysosome Function Causes Selective Cytotoxicity in VHL-Inactivated Renal Cell Carcinomas. Carcinogenesis 2020, 41, 828840, doi:10.1093/carcin/bgz161.

46. Neduva, V.; Russell, R.B. DILIMOT: Discovery of Linear Motifs in Proteins. Nucleic Acids Res. 2006, 34, W350-355, doi:10.1093/nar/gk1159.

47. Khacho, M.; Mekhail, K.; Pilon-Larose, K.; Pause, A.; Côté, J.; Lee, S. EEF1A Is a Novel 
Component of the Mammalian Nuclear Protein Export Machinery. Mol Biol Cell 2008, 19, 5296-5308, doi:10.1091/mbc.E08-06-0562.

48. $\quad$ Forbes, S.A.; Beare, D.; Boutselakis, H.; Bamford, S.; Bindal, N.; Tate, J.; Cole, C.G.; Ward, S.; Dawson, E.; Ponting, L.; et al. COSMIC: Somatic Cancer Genetics at High-Resolution. Nucleic Acids Research 2016, doi:10.1093/nar/gkw1121.

49. Rousseau, A.; Bertolotti, A. Regulation of Proteasome Assembly and Activity in Health and Disease. Nat Rev Mol Cell Biol 2018, 19, 697-712, doi:10.1038/s41580-018-0040-Z.

50. Hergovich, A.; Lisztwan, J.; Barry, R.; Ballschmieter, P.; Krek, W. Regulation of Microtubule Stability by the von Hippel-Lindau Tumour Suppressor Protein PVHL. Nat Cell Biol 2003, 5, 64-70, doi:10.1038/ncb899.

51. Grote, M.; Wolf, E.; Will, C.L.; Lemm, I.; Agafonov, D.E.; Schomburg, A.; Fischle, W.; Urlaub, H.; Lührmann, R. Molecular Architecture of the Human Prp19/CDC5L Complex. Mol Cell Biol 2010, 30, 2105-2119, doi:10.1128/MCB.01505-09.

52. Liu, Y.; Woods, N.T.; Kim, D.; Sweet, M.; Monteiro, A.N.A.; Karchin, R. Yeast Two-Hybrid Junk Sequences Contain Selected Linear Motifs. Nucleic Acids Res 2011, 39, e128, doi:10.1093/nar/gkr600.

53. Roberts, G.G.; Parrish, J.R.; Mangiola, B.A.; Finley, R.L. High-Throughput Yeast Two-Hybrid Screening. Methods Mol Biol 2012, 812, 39-61, doi:10.1007/978-1-61779-455-1_3.

54. Carmell, M.A.; Girard, A.; van de Kant, H.J.G.; Bourc'his, D.; Bestor, T.H.; de Rooij, D.G.; Hannon, G.J. MIWI2 Is Essential for Spermatogenesis and Repression of Transposons in the Mouse Male Germline. Dev Cell 2007, 12, 503-514, doi:10.1016/j.devcel.2007.03.001.

55. Sivagurunathan, S.; Palanisamy, K.; Arunachalam, J.P.; Chidambaram, S. Possible Role of HIWI2 in Modulating Tight Junction Proteins in Retinal Pigment Epithelial Cells through Akt Signaling Pathway. Mol Cell Biochem 2017, 427, 145-156, doi:10.1007/s11010-016-2906-8.

56. Henaoui, I.S.; Jacovetti, C.; Guerra Mollet, I.; Guay, C.; Sobel, J.; Eliasson, L.; Regazzi, R. PIWIInteracting RNAs as Novel Regulators of Pancreatic Beta Cell Function. Diabetologia 2017, 60, 1977-1986, doi:10.1007/s00125-017-4368-2.

57. Strub, G.M.; Paillard, M.; Liang, J.; Gomez, L.; Allegood, J.C.; Hait, N.C.; Maceyka, M.; Price, M.M.; Chen, Q.; Simpson, D.C.; et al. Sphingosine-1-Phosphate Produced by Sphingosine Kinase 2 in Mitochondria Interacts with Prohibitin 2 to Regulate Complex IV Assembly and Respiration. FASEB J 2011, 25, 600-612, doi:10.1096/fj.10-167502.

58. Peña, F.J.; Ortiz-Rodríguez, J.M.; Gaitskell-Phillips, G.L.; Gil, M.C.; Ortega-Ferrusola, C.; MartínCano, F.E. An Integrated Overview on the Regulation of Sperm Metabolism (Glycolysis-Krebs CycleOxidative Phosphorylation). Animal Reproduction Science 2021, 106805, doi:10.1016/j.anireprosci.2021.106805.

59. Vasudev, N.S.; Sim, S.; Cairns, D.A.; Ferguson, R.E.; Craven, R.A.; Stanley, A.; Cartledge, J.; Thompson, D.; Selby, P.J.; Banks, R.E. Pre-Operative Urinary Cathepsin D Is Associated with Survival in Patients with Renal Cell Carcinoma. Br J Cancer 2009, 101, 1175-1182, doi:10.1038/sj.bjc.6605250. 60. Young, A.C.; Craven, R.A.; Cohen, D.; Taylor, C.; Booth, C.; Harnden, P.; Cairns, D.A.; Astuti, D.; Gregory, W.; Maher, E.R.; et al. Analysis of VHL Gene Alterations and Their Relationship to Clinical Parameters in Sporadic Conventional Renal Cell Carcinoma. Clin. Cancer Res. 2009, 15, 7582-7592, doi:10.1158/1078-0432.CCR-09-2131.

61. Gossage, L.; Eisen, T. Alterations in VHL as Potential Biomarkers in Renal-Cell Carcinoma. Nat Rev Clin Oncol 2010, 7, 277-288, doi:10.1038/nrclinonc.2010.42.

62. Schuster, S.L.; Hsieh, A.C. The Untranslated Regions of MRNAs in Cancer. Trends Cancer 2019, 5, 245-262, doi:10.1016/j.trecan.2019.02.011. 